\title{
Influence of solar and geomagnetic activity on the ionosphere over Bulgaria
}

\author{
Bojilova R., Mukhtarov P. \\ National Institute of Geophysics, Geodesy and Geography NIGGG - BAS, Sofia, Bulgaria \\ E mail (bojilova@geophys.bas.bg).
}

Accepted: 10 October 2017

\begin{abstract}
An analysis of the seasonal dependence of the ionospheric response to the geomagnetic activity and the shortterm variations of the solar radiation has been made. The research is based on the critical frequency of the F-region measurements from the ionosonde station - Sofia for the period from 1995 to 2014. The crosscorrelations and delay times between the relative values of the critical frequencies of the ionospheric F-region with the planetary geomagnetic activity Kp-index and the solar radio flux at $10.7 \mathrm{~cm}$ (F10.7) for each calendar month of the year are investigated. The results can be used to create empirical models of the ionospheric parameters which depend on the variations of short-term solar and geomagnetic activity.
\end{abstract}

(c) 2018 BBSCS RN SWS. All rights reserved

Keywords: geomagnetic activity, solar activity, ionosphere, critical frequencies, cross correlation.

\section{Introduction}

In the present paper an attempt was made to determine the summarized characteristics of the response of the maximum electron density of the ionosphere under the influence of geomagnetic anomalies and short periodic variations of the solar ionizing radiation. The main focus is on determining the seasonal dependencies of these responses. A crosschecking analysis was used to determine not only the degree of interconnection of solar and ionospheric processes, but also the delay time of the ionosphere response.

The seasonal dependence of the geomagnetic response of the medium latitude ionosphere is due to the seasonal change in the dynamic mode of the atmosphere. Under the influence of the solar plasma flows into the polar oval, the neutral air temperature increases and the atomic oxygen / nitrogen (O / N2) ratio changes, which directly affects the rate of recombination. Heated air is transferred from the meridional component of the wind from high to medium latitudes and leads to negative ionospheric anomalies (a decrease in electron density), which is the most typical manifestation of ionospheric anomalies of geomagnetic origin in summer and equinox months (Prölss, 1995).

Variations of the solar ionizing radiation cause positive anomalies in the ionosphere. The changes in electron density within the 11-year solar cycle are not the subject of the present study. Variations in solar activity with a time scale of less than one month are considered. Both with high and low solar activity, such variations are observed in connection with the rotation of the Sun around its axis with a period of about 27days.

\section{Data}

The values for the planetary index of geomagnetic activity $\mathrm{Kp}$ are obtained from NOAA https://www.ngdc.noaa.gov. The data from the vertical sounding of the ionosphere with radio waves
(foF2) are from lonsospheric Station Sofia at NIGGGBAS.

The values of the solar F10.7 radio emission flux are obtained from NASA-https://omniweb.gsfc.nasa.gov/.

The used ionospheric, solar and geomagnetic data are processed according to the goals of the present study. From the measured values of the critical frequencies of the ionospheric F-region (foF2) (which are related to the maximum electron concentration with the dependence $\mathrm{Nm}\left[\mathbf{c m}^{-2}\right]=1,24.10^{4} \mathrm{foF}^{2}[\mathrm{MHz}]$ ) Relative deviations from the stationary diurnal course are calculated (Kutiev, I. and P. Muhratov, 2001).

$$
f o F 2_{r e l}(t)=\frac{f o F 2(t)-f o F 2_{m}(U T)}{f o F 2_{m}(U T)}
$$

Stationary 24-hour values are obtained by averaging the critical frequency values over a 31-day period centred on the current day for the same universal time that is in the current hour $t$. In this way, the diurnal, seasonal and solar dependence of foF2 is filtered out.

Values of F10.7 are processed in a similar manner:

$$
F 107_{\text {rel }}=\frac{F 107-F 107_{m}}{F 107_{m}} .
$$

The mean values of F10.7 (F10.7m) were obtained by averaging a 31-day running segment centred on the current day.

In this study are used filtered values- Kpf of the planetary geomagnetic index Kp:

$$
K p_{f}(t)=K p(t)-K p_{m}
$$

where $\mathrm{Kpm}$ is a mean value, obtained by averaging a 31-day running segment centred on the current day. Removing of running average value remove mean level of the geomagnetic activity, which correlate with stationary 24-hour values of the foF2.

Two examples illustrating the purpose of the present study are shown on Fig. 1. The left panel shows the course of the $\mathrm{Kp}$ index during a geomagnetic storm in August 2011 and the relative critical frequency. The negative ionosphere response as well as the delay and 
the geomagnetic anomaly are clearly visible. The right panel shows the solar activity fluctuation in March 2011 and the relative critical frequency (smoothed by threeday running averages). There is a positive response of the ionosphere also with some delay.

\section{Crosscorrelation analysis}

In present study normalized crosscorrelation function is obtained by following formula:

$\rho_{x y}(\tau)=\frac{M[x(t) y(t+\tau)]-M[x(t)] M[y(t)]}{\sqrt{M\left[x^{2}(t)\right]-M[x(t)]^{2}} \sqrt{M\left[y^{2}(t)\right]-M[y(t)]^{2}}}$, where $M$ is an operator to obtaining a mean value, $\tau$ is time lag.

The calculations of normalized crosscorrelation functions between solar and geomagnetic indexes and relative critical frequencies are based on the values for each calendar month of the years from 1995 to 2014. The days with average Kp $>4$ were excluded when calculating correlations with F107rel. Table 1 shows the number of days excluded from the calculation by months and their percentage relative to the total number of days.

Table 1.

\begin{tabular}{|c|c|c|c|c|c|c|c|c|c|c|c|c|}
\hline month & Jan & Feb & Mar & Apr & May & Jun & Jul & Aug & Sep & Oct & Nov & Dec \\
\hline days & 18 & 19 & 28 & 37 & 35 & 18 & 23 & 24 & 34 & 47 & 35 & 11 \\
\hline$\%$ & 2.9 & 3.4 & 4.5 & 6.2 & 5.6 & 3 & 3.7 & 3.9 & 5.7 & 7.6 & 5.8 & 1.8 \\
\hline
\end{tabular}
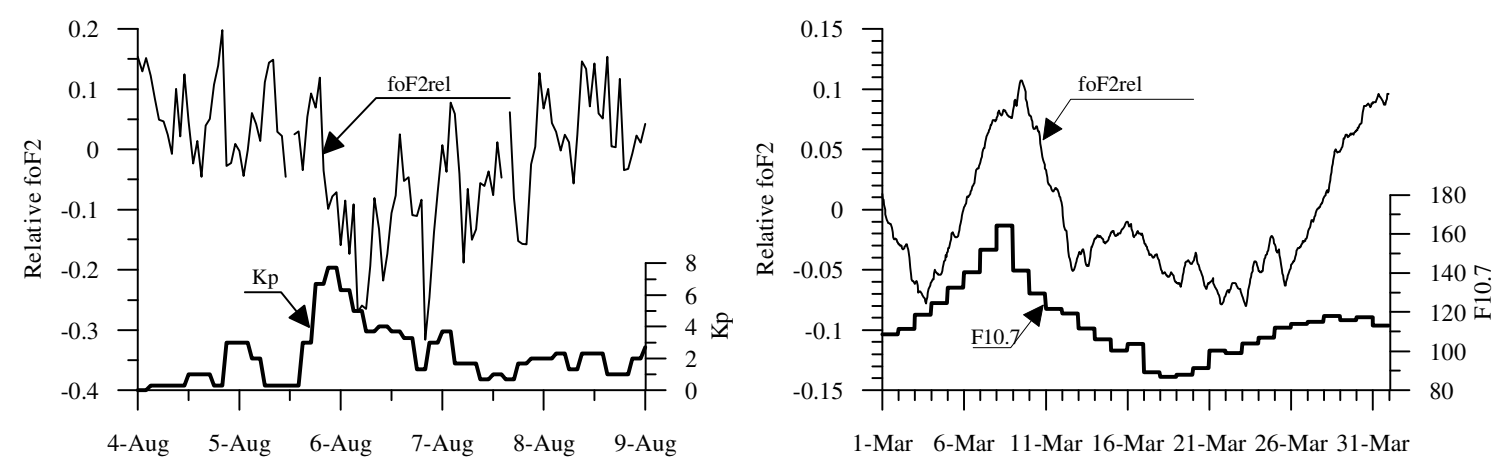

Fig. 1. Geomagnetic storm in August 2011 and solar variation in March 2011.

Fig. 2 shows the results for typical winter, spring, summer and autumn months. The croscorelation functions with the filtered geomagnetic index Kpf for the months of January and November indicate the presence of two types of response - positive with little delay and negative with significant delay.

From the presence of a positive and negative response of the ionosphere it can be concluded that two physical mechanisms of ionospheric response appear in the winter months (Andonov et al., 2011; Mukhtarov et al., 2013). In the summer, the Kpf correlations show only a negative response, with significantly less delay- about 12 hours. Correlations with the solar activity are positive, showing a longer delay-about 72 hours in the winter months.

Fig. 3 shows the seasonal course of the maximal negative values of the correlation functions and their corresponding time delays. The negative response of the geomagnetic disturbances is strong during the summer months (high negative correlations) with a little delay in time and decreases in the winter months with the delay increasing. The ionospheric response to variations in solar activity increases during the summer months compared to winter. However, the delay does not show a pronounced seasonal course.

The positive response of the relative critical frequency to the geomagnetic activity is observed only in the winter months from November to February. Fig. 4 shows the maximum correlations and their corresponding time delays. The maximum positive correlations are 0.1-0.2, and the time delay is practically the same 11-12 hours. The delay of the positive response is much shorter than that of the negative response in the same months while the maximum correlation values are of the same order as those of the negative response. 

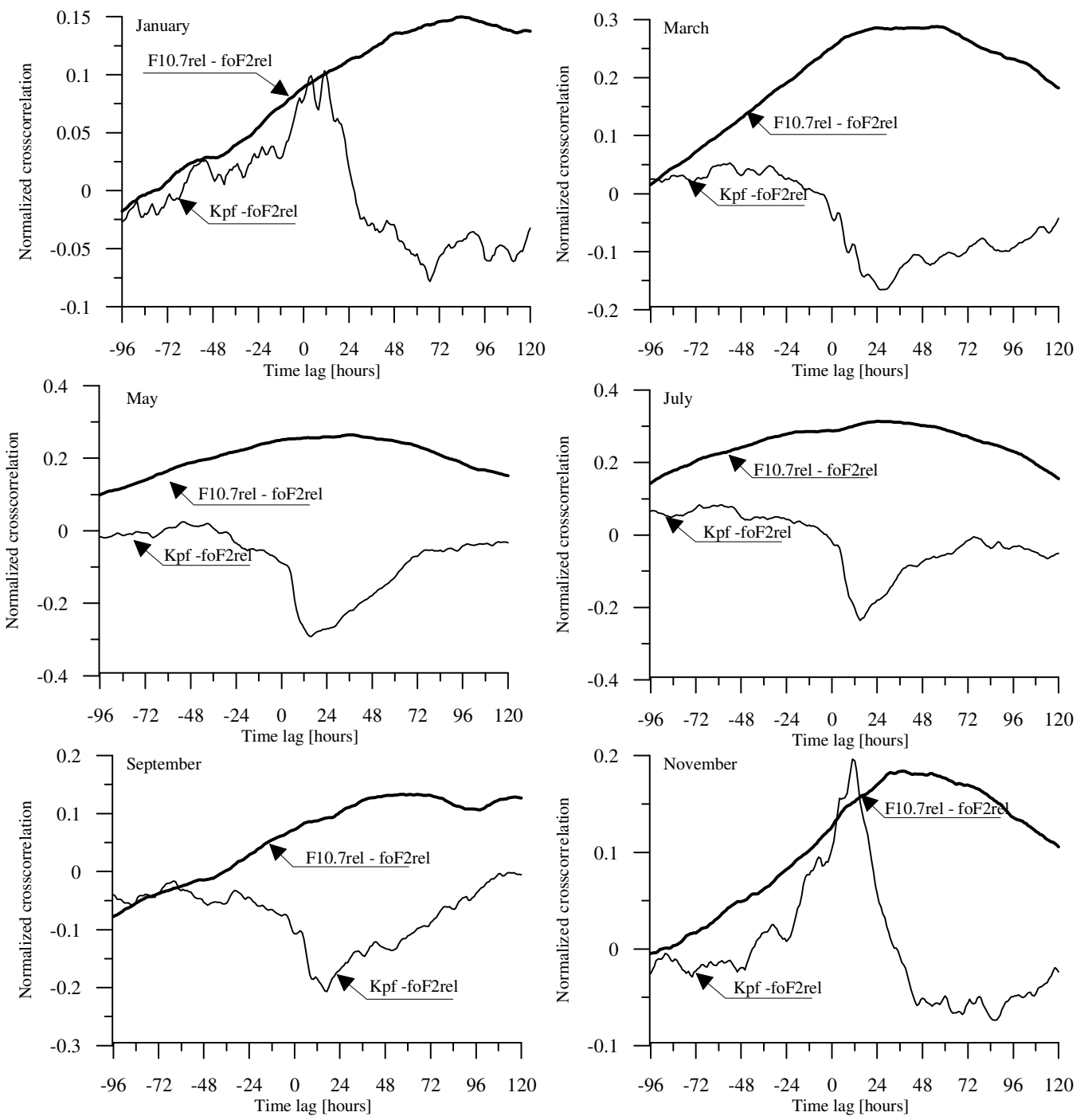

Fig. 2. Normalized crosscorrelation functions between relative foF2 and relative solar and filtered geomagnetic indices.
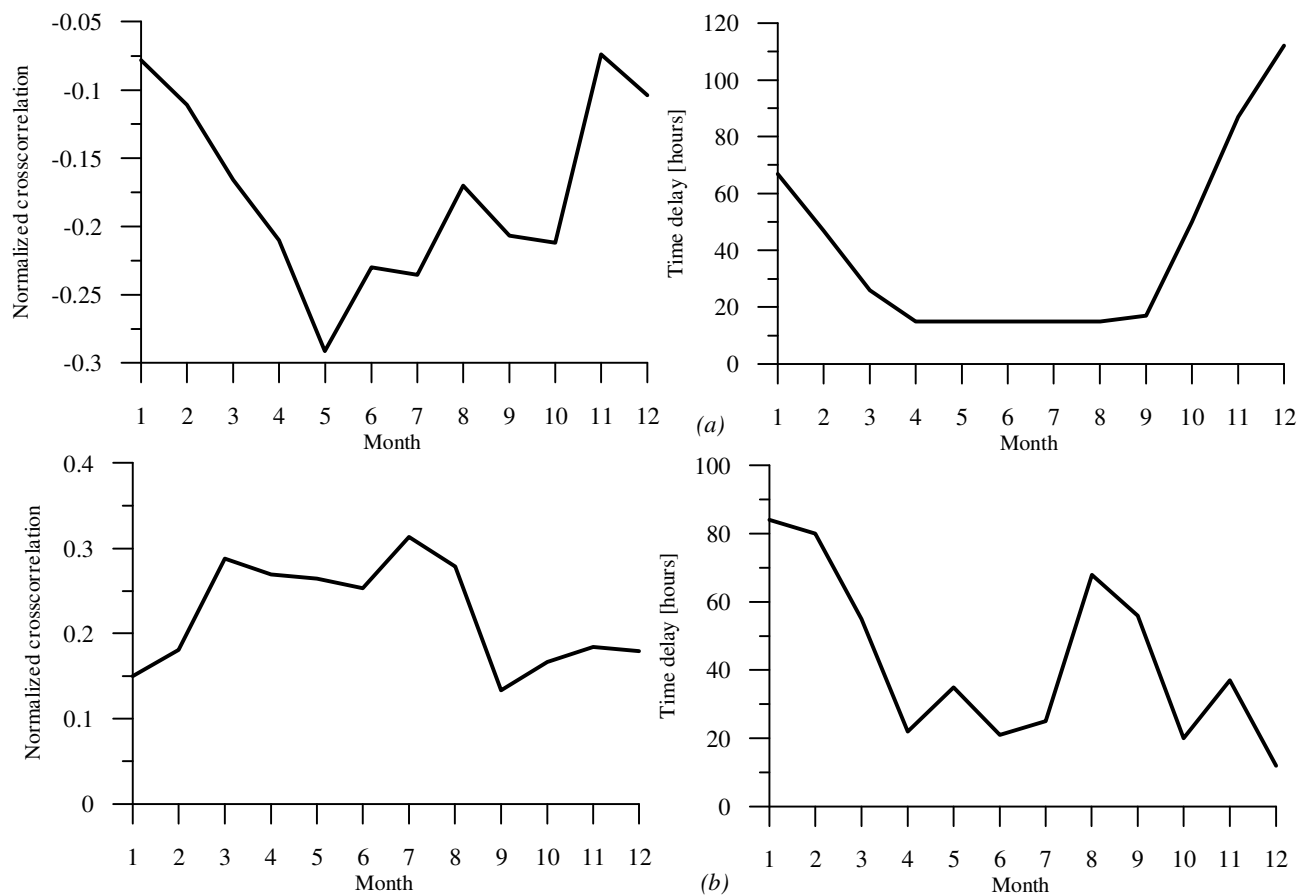

Fig. 3 (a) Seasonal variability of the maximum cross-correlation coefficients between the relative foF2 and filtered geomagnetic Kpindex (left plot) at negative ionospheric response and the corresponding time delay in hours (right plot);

3 (b) The same as (a) but the cross-correlation is between the relative foF2 and the relative solar F10.7and the time delay is in days. 

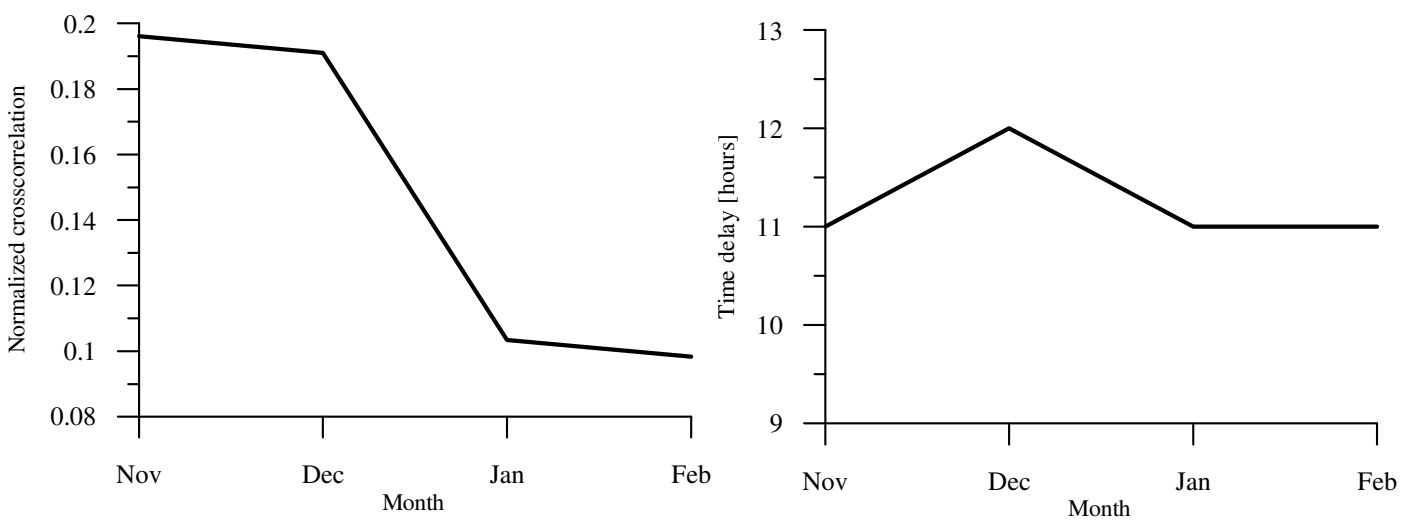

Fig. 4. Monthly variability of the maximum crosscorrelation coefficients between the relative foF2 and filtered geomagnetic Kp-index (left plot) at positive ionospheric response and the corresponding time delay in hours (right plot).

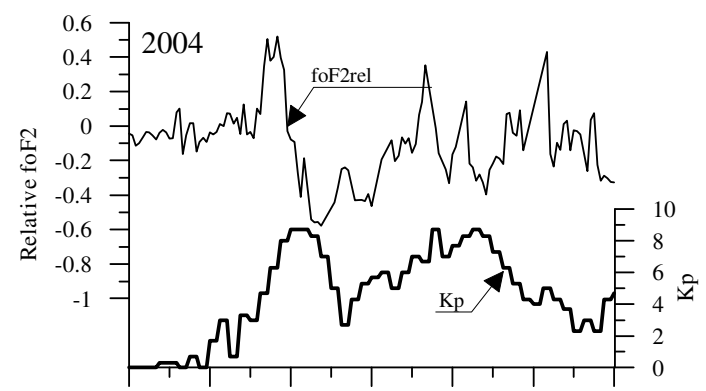

6-Nov 7-Nov 8-Nov 9-Nov 10-Nov 11-Nov 12-Nov

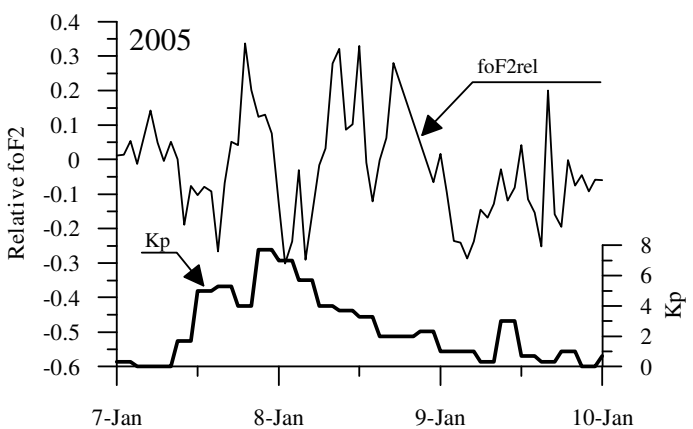

Fig. 5. The 3-hourly $\mathrm{Kp}$-index and the temporal variability of the relative foF2 for the period of time 7-12 November 2004 (left plot) and 7- 10 January 2005 (right plot).

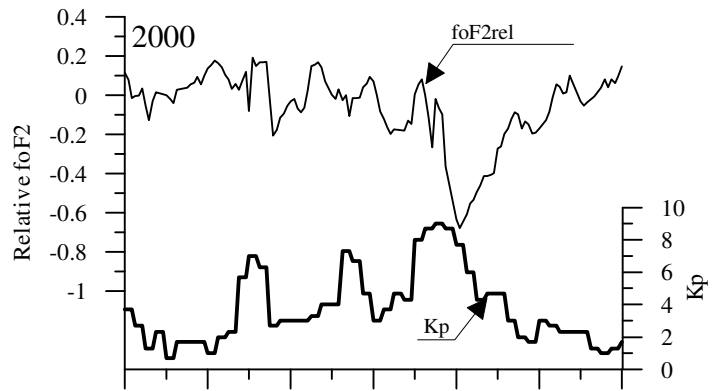

12-Jul 13-Jul 14-Jul 15-Jul 16-Jul 17-Jul 18-Jul

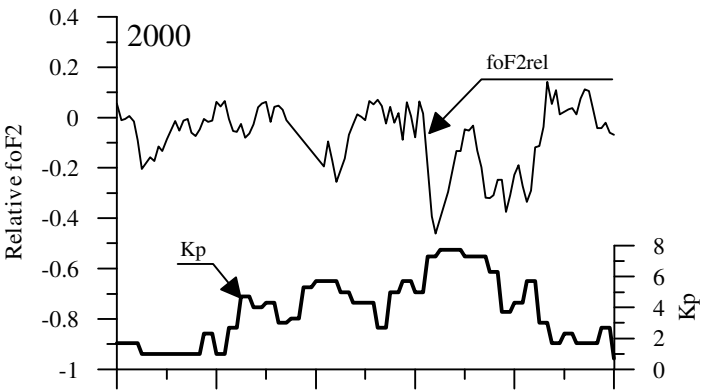

9-Aug 10-Aug 11-Aug 12-Aug 13-Aug 14-Aug

Fig. 6. The 3-hourly Kp-index and the temporal variability of the relative foF2 for the period of time 12-18 July 2000 (left plot) and 9-14 August 2000 (right plot).

The left plot of Fig. 5 shows the temporal variability of the relative critical frequencies during a typical winter geomagnetic storm in November 2004. The storm begins with a sudden positive response on 7 November which is followed by the negative response on 8 November. In this case two types of response are present during the storm - negative and positive. Significant positive and negative anomalies are also observed during the storm 7-8 January 2005 (right plot). A sustained negative response is observed on 9 January after the storm has ended. The examples illustrate the complexity of the critical frequency response to the geomagnetic storms during winter.

Fig. 6 illustrates typically temporal variability of the relative foF2 during geomagnetic storms in summer season. Positive anomalies are not detected (see cross correlation functions on Fig. 2). Negative anomalies occur with time delay.

\section{Conclusion}

The performed cross-correlation analysis between the filtered geomagnetic activity Kp-index and the critical frequency of the ionospheric F-region indicated the presence of a pronounced seasonal dependence of the ionospheric response. It has been found that during the summer months the ionospheric response is a negative one with a short delay time, around half a day. The observed summer negative ionospheric response is caused by the same directions of the disturbed and seasonal summer-to-winter circulations which are both equatorward. The circulation carries the molecular-rich air to midlatitudes that determines the increase of the loss rate at middle latitudes, i.e. defines the negative response of the foF2 (Penov et al., 2015).

Two types of response have been found in winter months; a positive response with a time delay of 
$\sim 12$ hours and a following negative response with longer time delay ( 50-1 10 hours). In winter hemisphere the summer-to-winter trans-equatorial thermospheric wind acts against the storm-time generated disturbed equatorward flow in winter. Then a circulation cell between high and middle latitudes is formed with upwelling flow at high latitudes and downwelling one at middle latitudes. The downwelling flow brings air rich in oxygen atoms. This means that the loss rate decreases at middle latitudes, i.e. the foF2 increases and that determines the positive response of the ionosphere with short delay time. However with the development of the geomagnetic storm the generated disturbed equatorward flow becomes significantly stronger than the seasonal summer-towinter circulation and brings heavy molecular constituents which lead to the decrease of the foF2 (Mukhtarov and Pancheva, 2012).

The ionospheric response to short-term F10.7 variability is always positive. Generally the delay time does not reveal a pronounced seasonal course except that the delay times in January and February ( 80 hours) are longer than those in April-July ( 40 hours).

\section{References}

Andonov, B., Mukhtarov, P., and Pancheva, D.: 2011, Advances in Space Research 48.6, 1041-1048.

Kutiev, I and Muhtarov, P.: 2013, Journal of geophysical research 106.A8, 15501-15510.

Mukhtarov, P., Andonov, B., and Pancheva, D.: 2013, Journal of Geophysical Research: Space Physics 118.10 , 6666-6685.

Mukhtarov, P. and Pancheva, D.: 2012, Journal of Atmospheric and Solar-Terrestrial Physics 90, 132-145.

Penov, N., Pancheva, D., Mukhtarov, P., Guerova, G.: 2015, Compt. rend. Acad. bulg. Sci, 68 (2), 241-250.

Prölss, G. W.: 1995, lonospheric F region storms, in Handbook for Atmospheric Electrodynamics, vol. 2, CRC Press, Boca Raton, pp. 195-248. 